\title{
RESEARCH
}

Open Access

\section{The CAD risk locus 9p21 increases the risk of vascular calcification in an iPSC-derived VSMC model}

Anja Trillhaase ${ }^{1,2,3}$, Beatrice Schmidt ${ }^{1,2,3}$, Marlon Märtens ${ }^{1,2,3}$, Undine Haferkamp ${ }^{4}$, Jeanette Erdmann ${ }^{1,2,3 \dagger}$ and Zouhair Aherrahrou ${ }^{1,2,3^{*}+}$ (D)

\begin{abstract}
Background: Coronary artery disease (CAD) is the leading cause of death worldwide. Chromosome locus 9p21 was the first to be associated with increased risk of CAD and coronary artery calcification (CAC). Vascular calcification increases the risk for CAD. Vascular smooth muscle cells (VSMCs) are one of the major cell types involved in the development of vascular calcification.
\end{abstract}

Methods: So far, mainly animal models or primary SMCs have been used to model human vascular calcification. In this study, a human in vitro assay using iPSC-derived VSMCs was developed to examine vascular calcification. Human iPSCs were derived from a healthy non-risk (NR) and risk (R) donor carrying SNPs in the 9p21 locus. Additionally, 9p21 locus knockouts of each donor iPSC line (NR and R) were used. Following differentiation, the iPSC-derived VSMCs were characterized based on cell type, proliferation, and migration rate, along with calcium phosphate (CaP) deposits. CaP deposits were confirmed using Calcein and Alizarin Red S staining and then quantified.

Results: The data demonstrated significantly more proliferation, migration, and CaP deposition in VSMCs derived from the $\mathrm{R}$ and both KO iPSC lines than in those derived from the NR line. Molecular analyses confirmed upregulation of calcification markers. These results are consistent with recent data demonstrating increased calcification when the 9p21 murine ortholog is knocked-out.

Conclusion: Therefore, in conclusion, genetic variation or deletion of the CAD risk locus leads to an increased risk of vascular calcification. This in vitro human IPSC model of calcification could be used to develop new drug screening strategies to combat CAC.

Keywords: Coronary artery disease, Vascular calcification, 9p21, iPSCs, VSMCs

\footnotetext{
* Correspondence: zouhair.aherrahrou@uni-luebeck.de

${ }^{\dagger}$ Jeanette Erdmann and Zouhair Aherrahrou contributed equally to this work.

${ }^{1}$ Institute for Cardiogenetics, University of Luebeck, Ratzeburger Allee 160, 23562 Luebeck, Germany

${ }^{2}$ DZHK (German Centre for Cardiovascular Research), Partner Site Hamburg/ Kiel/Luebeck, Luebeck, Germany

Full list of author information is available at the end of the article
}

(C) The Author(s). 2021 Open Access This article is licensed under a Creative Commons Attribution 4.0 International License, which permits use, sharing, adaptation, distribution and reproduction in any medium or format, as long as you give appropriate credit to the original author(s) and the source, provide a link to the Creative Commons licence, and indicate if changes were made. The images or other third party material in this article are included in the article's Creative Commons licence, unless indicated otherwise in a credit line to the material. If material is not included in the article's Creative Commons licence and your intended use is not permitted by statutory regulation or exceeds the permitted use, you will need to obtain permission directly from the copyright holder. To view a copy of this licence, visit http://creativecommons.org/licenses/by/4.0/ The Creative Commons Public Domain Dedication waiver (http://creativecommons.org/publicdomain/zero/1.0/) applies to the data made available in this article, unless otherwise stated in a credit line to the data. 


\section{Background}

Coronary artery disease (CAD) is the leading cause of death worldwide [1]. Atherosclerosis, one of the major complications of CAD, is caused by a buildup of plaque in the coronary arteries. Common risk factors for CAD include hyperlipidemia, hypertension, diabetes, obesity, and smoking, as well as increasing age $[2,3]$. Additionally, it is estimated that CAD has a heritability factor of up to $50 \%$ [4, 5]. Genome-wide association studies (GWAS) have identified 163 genetic loci that are significantly associated with CAD [6]. Among these, the 9p21 locus was one of the first identified [7] and has been replicated by additional studies [8-10]. Furthermore, five CAD risk genes (9p21, ADAMTS7, PHACTR1, MRAS, and COL4a1/COL4a2) are associated with coronary artery calcification (CAC) [11].

Vascular calcification is described as deposition of calcium phosphate $(\mathrm{CaP})$ minerals, usually as hydroxyapatite or inorganic phosphate (Pi), in cardiovascular tissues [12]. Vascular calcification is associated with a three- to four-fold increase in the risk of cardiovascular mortality [13], making it an important disease marker. One of the major cell types involved in the vascular calcification process are vascular smooth muscle cells (VSMCs). During calcification, VSMCs lose expression of SMCspecific markers and start expressing osteogenic genes [13-21], which results in the deposition of bone-like minerals such as $\mathrm{CaP}$ in the extracellular matrix (ECM) [13-15, 21].

Primary VSMCs are challenging to use because they lose their phenotypic properties when cultured in vitro, making it difficult to conduct long-term in vitro experiments with these cells. Additionally, they require characterization before they can be transplanted in vivo [22]. Therefore, induced-pluripotent stem cells (iPSCs) are a promising alternative to study vascular disease progression without the need of repetitive surgery to harvest primary VSMCs.

Human iPSCs can be generated from a variety of adult somatic cell types [23], and they provide an almost unlimited source of regenerative cells [23]. Human iPSCs can be differentiated into most cell types present in the adult human body [24], including VSMCs. However, there are only a few published methods for differentiating VSMCs from iPSCs. More recently, more complex protocols for generating human iPSC-derived VSMCs have been published that allow lineage-specific [25] or phenotype-specific [26] differentiation of VSMCs. Nevertheless, to date, there is no published protocol for differentiating iPSCs into calcifying vascular cells.

\section{Materials/methods}

\section{Cell culture}

Human iPSCs were reprogrammed from peripheral blood mononuclear cells (PBMCs) obtained from a healthy non-risk (HE463_7; NR) and a risk (C512, R) donor as previously described [27]; the latter carried SNPs in the 9p21 CAD risk interval. The authors of this previous study kindly provided the R, NR, and KO iPSC lines for the present study [27]. Human iPSCs of the four genotypes (R WT, NR WT, R KO, NR KO) were cultivated in mTeSR1 (StemCell Technologies) medium on Geltrex-coated dishes (Geltrex hESC qualified, readyto-use, GFR, Thermo Fisher). Medium was exchanged every day. Additionally, a healthy control cell line, UCSD018i-3-6 (WiCell; referred to as 18i-3-6), was used as internal control and cultured as mentioned above.

Differentiation of iPSC into lateral-mesoderm (LM)derived VSMCs was performed as described previously, with slight modifications [25]. Briefly, iPSCs were cultured in MTeSR1 and switched to maintenance medium (CDM-BSA) supplemented with $12 \mathrm{ng} / \mathrm{mL}$ FGF2 and $0.1 \mathrm{ng} / \mathrm{mL}$ Activin A for $1 \mathrm{~d}$. Next, the cells were replated (d0) at a lower cell density than used in the original protocol [25] because a 1:2 to $1: 3$ splitting ratio was found to improve cell viability during mesoderm differentiation. Pluripotent cells were differentiated into early mesoderm properties within $1.5 \mathrm{~d}$ by supplementation with $20 \mathrm{ng} / \mathrm{mL}$ FGF2-IS, $10 \mu \mathrm{M}$ LY294002, and 10 $\mathrm{ng} / \mathrm{mL}$ BMP4, as reported by Cheung and colleagues. Between $\mathrm{d} 1.5$ and $\mathrm{d} 5$, the cells were differentiated into LM cells by adding $20 \mathrm{ng} / \mathrm{mL}$ FGF2-IS and $0.5 \mathrm{ng} / \mathrm{mL}$ BMP4. On $\mathrm{d} 5$, the cells were replated according to the published protocol. However, cell viability was improved by completely replenishing the medium every second day instead of replacing only $50 \%$. From d5 until d18, the medium was supplemented with $10 \mathrm{ng} / \mathrm{mL}$ PDGF$\mathrm{BB}$ and $2 \mathrm{ng} / \mathrm{mL}$ TGF $\beta 1$ [25]. For all cell lines, except the NR WT line, cells had to be passaged at a 1:2 to $1: 3$ ratio between $\mathrm{d} 8$ and $\mathrm{d} 14$. In the NR WT line, we did not observe the necessity to passage during differentiation. The resulting VSMCs were cultured on $0.1 \%$ gelatin-coated flasks in SMC maintenance medium (450 mL DMEM with high Glutamax $+50 \mathrm{~mL}$ FCS Gold Plus $+5 \mathrm{~mL} \mathrm{Pen} /$ Strep; referred to as SMC medium). Medium was exchanged twice a week.

\section{Calcification}

For calcification, VSMCs derived from the R WT, R KO, and NR KO iPSC lines were replated onto gelatin-coated 24-well plates at a density of 25,000-35,000 cells/well, or 50,000-55,000 cells/well for NR WT-derived VSMCs. At approximately $85-90 \%$ confluency, the calcification protocol was initiated. SMC maintenance medium was supplemented with the calcifying agents, as previously reported [28]. Medium was exchanged every 2-3 days. After 7 days, the calcification protocol was terminated, and the cells were used for immunostaining and RNA extraction. 
Immunofluorescence (IF) staining

Detection and localization of the target proteins within cultured iPSCs or iPSC-derived VSMCs was performed by immunofluorescence staining. For IF analysis, cells were grown on $\mathrm{Nunc}^{\mathrm{Tm}}$ Lab-Tek $^{\text {TM }}$ II Chamber Slides ${ }^{\mathrm{Tm}}$ (Thermo Scientific, \#154534) at a seeding density of $0.5 \times 10^{4}$ to $2 \times 10^{4}$ cells per $\mathrm{cm}^{2}$. Cells were washed with PBS and then fixed for $30 \mathrm{~min}$ (mins) with chilled ($20^{\circ} \mathrm{C}$ ) methanol to acetone (1:1). A Dako Pen (Dako, \#S2002) was used to outline the wells to prevent antibody solutions from merging. For antibody staining, cells were permeabilized in PBS containing 0.1\% Triton-X100 (Sigma Aldrich, \#T-8787) and 1\% BSA, then blocked with PBS containing 3.5\% BSA. Next, $100 \mu \mathrm{L}$ of primary antibody diluted in PBS (1:300-1:500), or PBS alone (negative control), was applied to each well and incubated overnight at $4{ }^{\circ} \mathrm{C}$ (Table 1 ).

Slides were washed three times with PBS (each for 5 $\mathrm{min}$ ) and then incubated for $1 \mathrm{~h}$ at room temperature with a secondary antibody (1:300-1:500) diluted in PBS (Table 1). Secondary antibodies were conjugated to a fluorescent dye (Alexa Fluor ${ }^{\circ}$ ) for target protein detection.

Subsequently, cells were washed three times with PBS, each for $5 \mathrm{~min}$. To counterstain the nucleus of the cells, the blue-fluorescent stain 4',6-diamidino-2-phenylindole (DAPI) was used, which binds to double-stranded DNA. Slides were incubated in the dark for 15 min with $0.1 \mu \mathrm{g} /$ $\mathrm{mL}$ DAPI solution and washed with deionized water. The slides were covered using Dako Fluorescence mounting medium (Dako, \#S3023) and glass cover slips (Menzel, \#9161060). Detection of IF staining was performed using a BZ-9000 BioRevo ${ }^{\text {Tw }}$ fluorescence microscope (Keyence, Neu-Isenburg, Germany).

\section{Proliferation assay}

Cells were stained with the Ki67 antibody according to the IF protocol. Nuclei were counterstained with DAPI. DAPI+ and Ki67+ cells were counted and the proportion of Ki67+ cells was compared with the overall number of cells (DAPI+). Results are presented as the percentage of $\mathrm{Ki} 67+$ cells in at least three independent biological replicates.

\section{Migration assay}

For the scratch assay, VSMCs were seeded into a 12-well plate and cultured until confluent. Using a $100 \mu \mathrm{L}$ tip, the cell layer in each well was scratched vertically. A single phase-contrast image was taken at previously specified positions in each well using an Olympus IX70 inverted microscope and CellSens Software. After $24 \mathrm{~h}$, new images were taken at the same positions. Using the CellSens Software, the Confluency Checker tool was used to calculate the confluency at $0 \mathrm{~h}$ and $24 \mathrm{~h}$. All time points included in the experiment were processed and confluency was analyzed. The difference between the confluency of $0 \mathrm{~h}$ and $24 \mathrm{~h}$ was set equal to the migration rate in \%. Three independent biological replicates were analyzed.

\section{ARS and Calcein staining}

Calcium phosphate $(\mathrm{CaP})$ deposition was detected by Calcein (C30H26N2O13), a fluorescent chromophore that binds specifically to $\mathrm{Ca}^{2+}$ in cultured iPSC-VSMCs (Du et al., 2001). Cultured cells were washed once with PBS and fixed in chilled $\left(4{ }^{\circ} \mathrm{C}\right) 4 \%$ paraformaldehyde (PFA) for $30 \mathrm{~min}$. Cells were then incubated for $30 \mathrm{~min}$ in the dark with Calcein staining solution (Sigma, \#C0875), washed three times with $50 \mathrm{mM}$ TBS (pH 9) (each for $3 \mathrm{~min}$ ), and then counterstained with DAPI for $10 \mathrm{~min}$ in the dark. Cells were covered with $400 \mu \mathrm{L}$ of $50 \mathrm{mM}$ TBS (pH 9), and Calcein fluorescence was detected using a BZ-9000 BioRevoTM fluorescence microscope (Keyence, Neu-Isenburg, Germany) immediately after staining. Alizarin Red S (ARS) staining was performed using the osteogenesis quantitation kit (Merck Millipore, \# ECM815), following the manufacturers' instructions. Stained cells were imaged using the BZ-9000 BioRevo $^{\mathrm{Ts}}$ fluorescence microscope (Keyence, NeuIsenburg, Germany).

\section{Calcein quantification}

Using a python script (Appendix), kindly provided by Dr. Tobias Reinberger from the Institute for Cardiogenetics, Luebeck, DAPI and Calcein intensity, and the pixel count, were estimated. The Calcein intensity was

Table 1 Antibodies used for IF

\begin{tabular}{lllll}
\hline Antibody & Host species & Dilution & Manufacturer & Cat. number \\
\hline hCNN1 & Mouse & $1: 500$ & Sigma & C2687 \\
hKi67 (marker of proliferation Ki67) & Mouse & $1: 300$ & BD Pharmingen & Cell Signaling \\
hNANOG & Rabbit & $1: 300$ & Abcam & ab14106 \\
hTAGLN & Rabbit & $1: 500$ & Invitrogen & A21206 \\
Anti-rabbit lgG & Donkey & $1: 300$ & Invitrogen & A11029 \\
Anti-mouse lgG & Goat & $1: 500$ & Invitrogen & A10037 \\
Anti-mouse lgG & Donkey & $1: 500$ & &
\end{tabular}


then normalized to DAPI (Intensity Calcein/DAPI pixel count). Untreated VSMCs were used as a reference.

\section{RNA isolation and quantitative polymerase chain reaction} (qPCR)

mRNA was isolated from cells using the Qiagen RNEasy Mini Plus Kit (Qiagen, \#74136), including DNase I treatment, following manufacturers' instructions. RNA concentration and purity were determined using a BioPhotometer (Eppendorf, Hamburg, Germany), and samples were stored at $-80^{\circ} \mathrm{C}$ until required. Synthesis of complementary DNA (cDNA) was performed via reverse transcription as follows: $10 \mu \mathrm{L}$ of RNA, adjusted to a concentration of $100 \mathrm{ng} / \mu \mathrm{L}$ in DNase/RNase-free water (Gibco, 10977035), was incubated for $5 \mathrm{~min}$ at $68^{\circ} \mathrm{C}$. Next, $10 \mu \mathrm{L}$ of reaction mix for reverse transcription, containing $4 \mu \mathrm{L} 5 \times$ First Strand Buffer (Invitrogen, \#28025021), $2 \mu \mathrm{L}$ of $100 \mathrm{mM}$ dithiothreitol (DTT) (Invitrogen, \#28025021), and $1 \mu \mathrm{L}$ each of 2 '-deoxyribonucleosid-5' -triphosphates (dNTPs; $4 \mathrm{mM}$ ) (Promega, \#U1330), Random Hexamer Primer-Mix (Roth, \#HP28.1), RiboLock (40 U/ $\mu \mathrm{L})$ (Thermo Scientific, \#EO0381), and M-MLV RT (200 U/ $\mu \mathrm{l})$ (Invitrogen, 28025021) were added to the RNA and incubated for $1 \mathrm{~h}$ at $37^{\circ} \mathrm{C}$, followed by enzyme inactivation at $95^{\circ} \mathrm{C}$ for $5 \mathrm{~min}$. Finally, the cDNA was stored at $-20^{\circ} \mathrm{C}$ until further use. Quantitative gene expression analysis, known as PCR, was performed on cDNA using a $7900 \mathrm{HT}$
Fast Real-Time PCR System (Applied Biosystems, \#4329001) and the corresponding SDS 2.2.2 software.

\section{Statistical analyses}

All data points are represented as dot plots, unless otherwise stated. The median is represented by horizontal bars. Unpaired $t$ tests using Welch's correction were performed using GraphPad Prism 6.04. $P<0.05$ was considered statistically significant. For all analyses, $P$ values are indicated as significant $\left({ }^{*} P<0.05\right.$, ${ }^{* *} P<0.01$, ***P $P<0.001$, $* * * * P<0.0001$ ), or not significant (n.s.), unless stated otherwise. Statistic outliers were identified using the GraphPad outlier test and then excluded from the analyses.

\section{Results}

Differentiation of iPSCs into VSMCs and their characterization

Human iPSCs generated from a 9p21 risk (R) or a non-risk (NR) donor were differentiated into lateral mesodermderived VSMCs by slight modification of a previously published protocol (Cheung et al., 2014).

The undifferentiated iPSCs and their derived VSMCs were characterized based on morphology, where iPSCs show a typical colony growth with tightly packed cells, while VSMCs look star or stellate shaped. Further, RNA profile, protein expression, and localization were analyzed in both cell types. Phase contrast imaging revealed

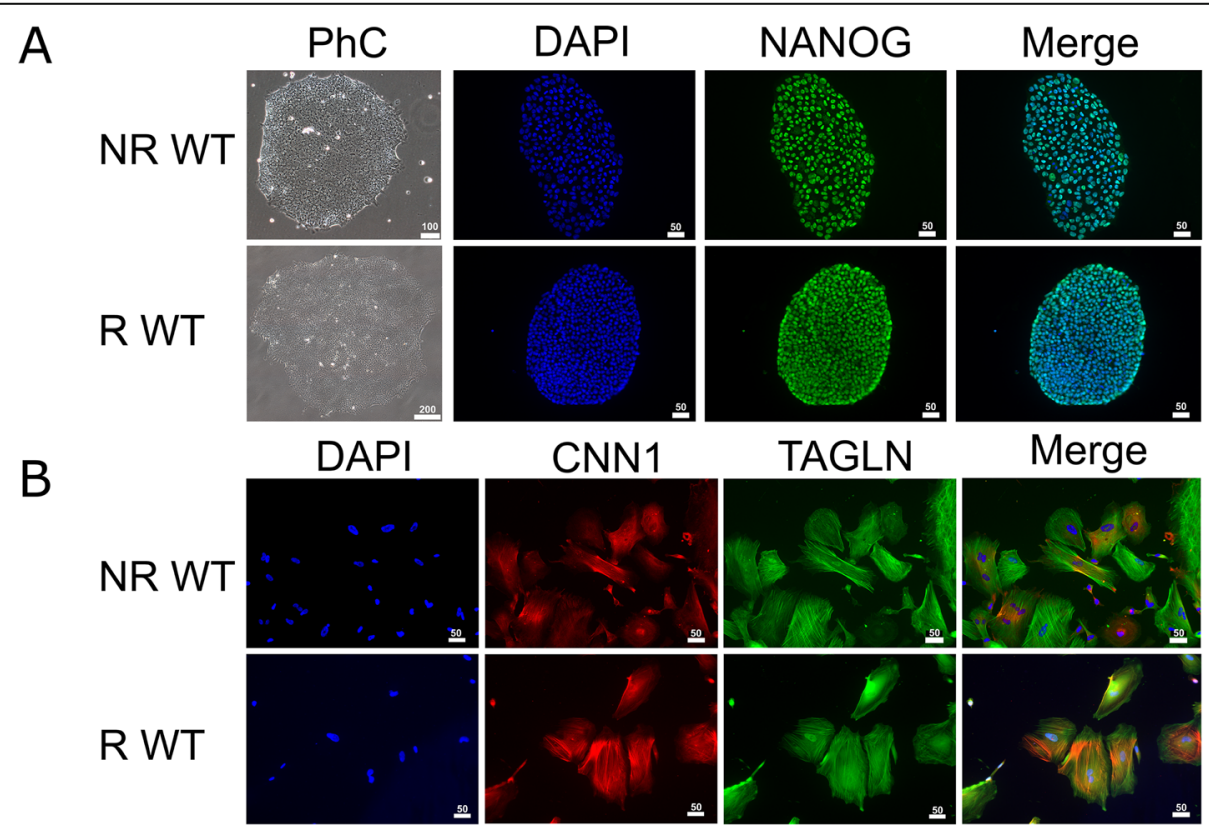

Fig. 1 Characterization of WT iPSCs and derived VSMCs. a NR (top) and R (bottom) WT iPSCs show similar iPSC morphology, as seen in the phase contrast (PhC) images. Scale bars represent $100 \mu \mathrm{m}$. Staining with the pluripotency marker NANOG (green) confirmed nuclear expression, as seen in the overlaid image (merge). Nuclei were counterstained with DAPI (blue). Scale bars represent $50 \mu \mathrm{m}$. b NR (top) and R (bottom) WT iPSC-derived VSMCs show colocalization of CNN1 (red) and TAGLN (green) in the contractile apparatus of the cells. No differences were observed between R and NR WT VSMCs. Nuclei were counterstained with DAPI. Scale bars represent $50 \mu \mathrm{m}$ 
that the NR and R WT undifferentiated iPSCs had similar morphology (Fig. 1). Immunofluorescence (IF) staining with the pluripotency marker NANOG and nuclear co-stain DAPI showed clear nuclear protein localization (Fig. 1a). Overall, there were no differences in morphology or protein expression between the NR or R WT iPSCs. The 9p21 CAD risk locus was knocked-out from both $R$ and NR donor iPSC lines (9p21 R and NR KO), as previously described [27] and then characterized using the same parameters. Both the 9p21 R and NR KO lines displayed typical morphologies, with identical nuclear localization of NANOG protein (Suppl. Figure 1).

The iPSC-derived VSMCs were characterized based on their morphology, protein expression, and protein localization. IF staining of CNN1 and TAGLN, two common SMC markers, revealed co-expression in the contractile fibers of iPSC-derived VSMCs. This was observed in both the NR- and R WT-derived VSMCs (Fig. 1b). No significant morphological or protein expression differences were observed between $\mathrm{R}$ and NR
WT cells. Similarly, the 9p21 KO iPSC-derived VSMCs displayed normal morphology, and colocalization of CNN1 and TAGLN in the contractile fibers (Suppl. Figure 2). No differences were detected between the KOs from the R or NR donors.

Additionally, expression of mRNAs encoding pluripotency- and SMC-specific genes was compared across all cell lines. The expression of NANOG, OCT4, and SOX2, were significantly decreased in NR and R WT derived VSMCs, compared to undifferentiated iPSCs (Fig. 2a). There were no significant differences between the $R$ and NR cell lines. Expression of mRNAs encoding SMCassociated genes TAGLN, CNN1, and CALD1 was significantly higher in R- and NR WT-derived VSMCs than in undifferentiated iPSCs (Fig. 2b). NR KO- and R KOderived VSMCs showed lower expression of pluripotency markers than iPSCs (Fig. 2c), while SMC markers were significantly upregulated in VSMCs compared with iPSCs (Fig. 2d). Overall, no significant differences in gene expression were observed between the genotypes. To validate

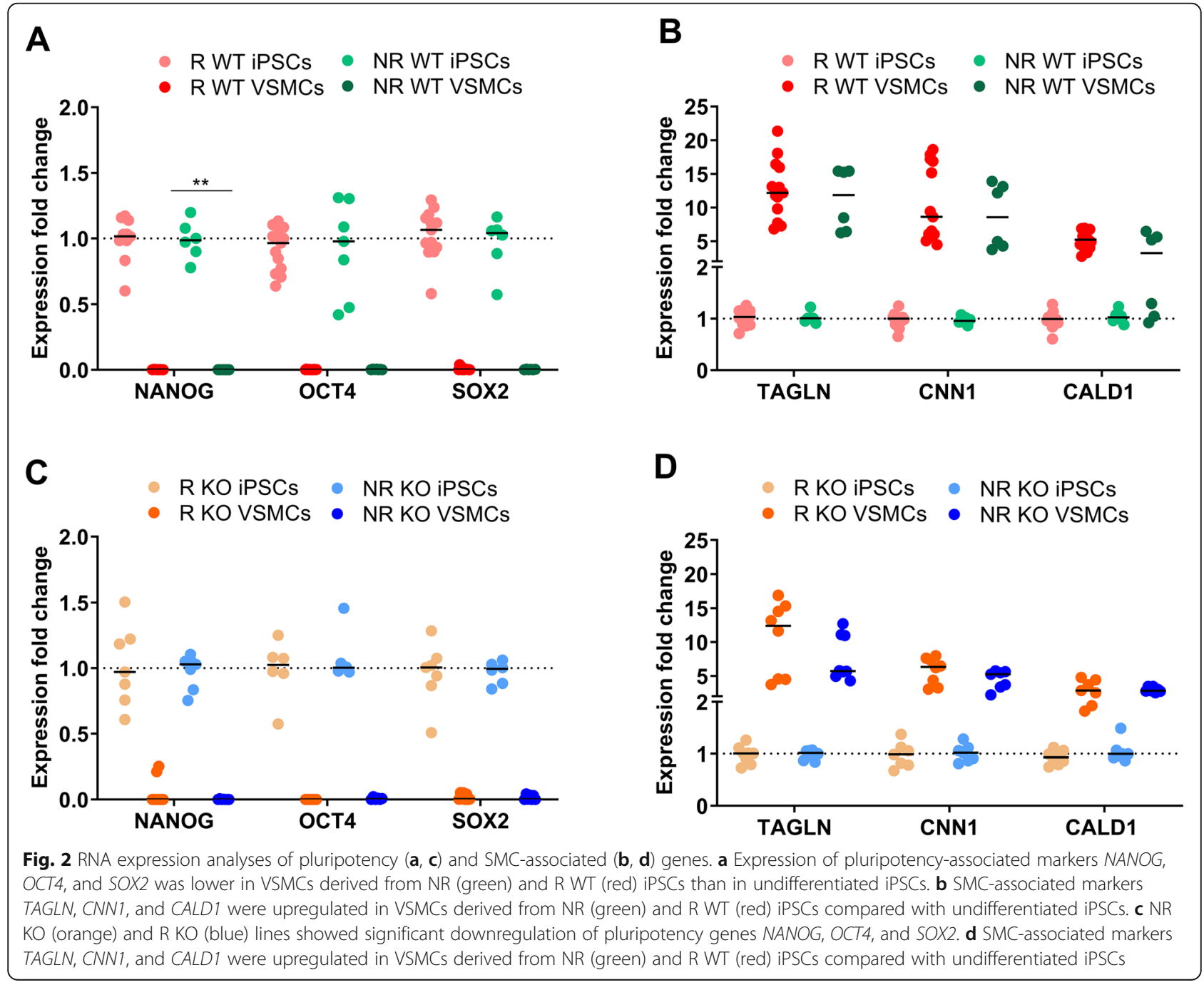


the RNA results, we performed western blot analysis to detect expression of OCT4 and TAGLN proteins (data not shown).

\section{9p21 affects iPSC-derived VSMC proliferation and migration}

To further analyze iPSC-derived VSMCs, we examined their proliferation, migration, and calcification properties.

First, to estimate the number of proliferative cells, Ki67 IF staining was performed. R WT VSMCs contained a higher amount of Ki67 positive $(+)$ cells than NR WT VSMCs (Fig. 3a; Suppl. Figure 3). R KO VSMCs also contained more Ki67+ cells than NR KO cells (Fig. 3b; Suppl. Figure 4). Together, these results indicated increased proliferation of R donor iPSCs. Furthermore, the migratory capacity of iPSC-derived VSMCs was characterized in a scratch assay. R WT VSMCs showed a significantly higher migration rate than NR WT VSMCs (Fig. 3c; Suppl. Figure 5). However, there were no differences in migration rate between $\mathrm{R}$ and NR KO iPSCderived VSMCs (Fig. 3d; Suppl. Figure 6).
Testing and optimizing calcification protocols with iPSCderived VSMCs

To identify the calcification protocol that was most suitable for our aim, we tested different calcification methods. First, the most common calcification method was tested, which involves supplementation with $\mathrm{Pi}$ or a combination of betaglycerophosphate ( $\beta$-GP) and L-ascorbic acid phosphate (LAP). However, this did not result in positive calcification (data not shown). Next, the commercial StemXVivo ${ }^{\circ}$ human osteogenic medium (R\&D Systems, \#CCM008/ CCM007) was tested for 30 days. The initial trials in one cell line looked quite promising; however, when all cell lines were tested the calcification efficiency was only $20 \%$. Next, the StemXVivo ${ }^{\circ}$ medium was supplemented with TNF $\alpha$, which achieved $25 \%$ calcification efficiency. Additionally, various combinations of $\mathrm{H}_{2} \mathrm{O}_{2}$ were combined with $\mathrm{BMP} 2$ and TNFa. However, $\mathrm{H}_{2} \mathrm{O}_{2}$ led to extensive apoptosis of iPSC-derived VSMCs, and addition of TNF $\alpha$ and BMP2 did not induce calcification. Next, two recently published protocols for in vitro calcification of human and mouse VSMCs were compared. The Alves and colleagues (2014) calcification cocktail comprised $0.1 \mathrm{mML}$-AP, $10 \mathrm{mM} \beta$ -

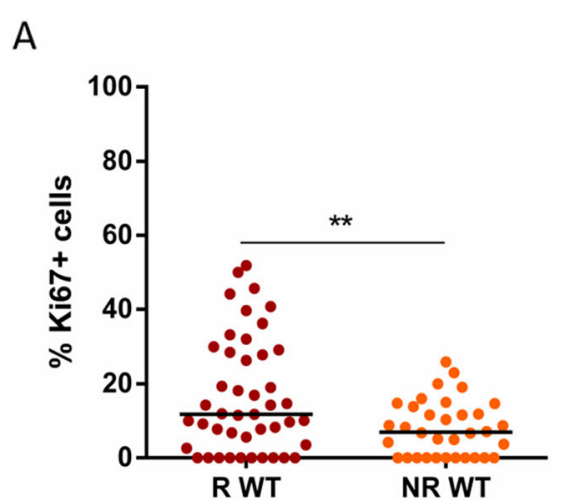

B
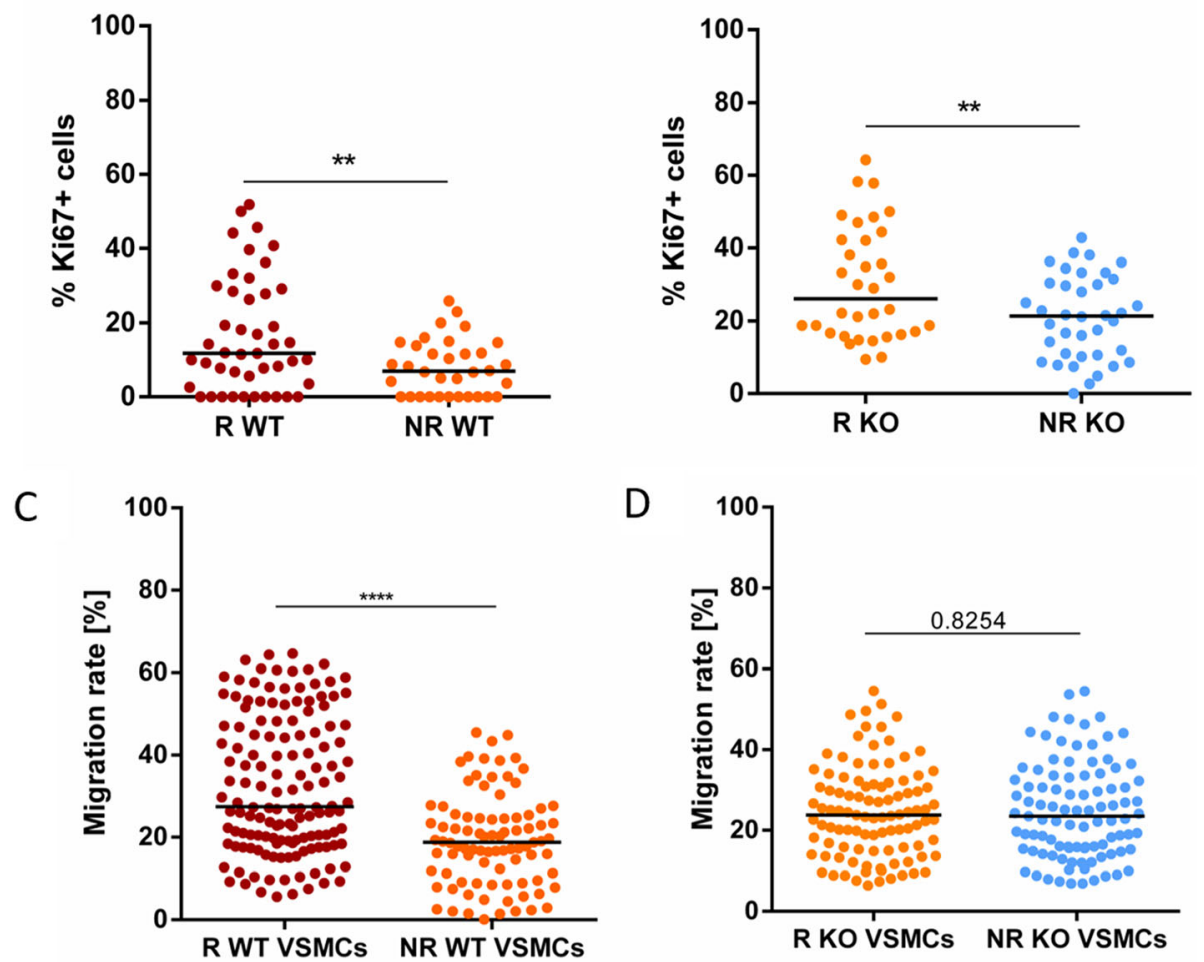

Fig. 3 The 9p21 locus influences proliferation and migration of iPSC-derived VSMCs. a R WT (red) derived VSMCs showed significantly higher proliferation rates than those derived from NR WT (orange). $\mathbf{b}$ R KO (orange) VSMCs showed higher proliferation rates than those derived from NR KO (blue). c R WT (red) VSMCs showed significantly higher migration rates than those derived from NR WT (orange). $\mathbf{d}$ There were no differences in migration rate between $\mathrm{R}$ KO (orange) and NR KO (blue) VSMCs 
GP, and $100 \mathrm{nM}$ dexamethasone in either SMC or StemX$\mathrm{Vivo}^{\circ}$ medium containing $1.8 \mathrm{mM}$ calcium chloride $\left(\mathrm{CaCl}_{2}\right)$ and $20 \mathrm{mM}$ HEPES [29]. When tested, this cocktail did not result in detectable calcification of iPSC-derived VSMCs. The final protocol comprised $4 \mathrm{mM} \mathrm{CaCl}, 5 \mathrm{mM} \beta-\mathrm{GP}$, $50 \mu \mathrm{g} / \mathrm{ml} \mathrm{L}-\mathrm{AP}, 1 \mu \mathrm{M}$ insulin, and $0.1 \mu \mathrm{M}$ dexamethasone in StemXVivo ${ }^{\bullet}$ medium for $7 \mathrm{~d}$ [28]. This protocol achieved an overall calcification efficiency of almost 40\% (Suppl. Figure 7). Based on these tests, the Tziakas method (referred to as Tziakas cocktail) was chosen for further calcification experiments.

\section{Successful calcification of VSMCs derived from R and KO iPSCs}

Characterized iPSC-derived VSMCs were calcified by incubation for 7 days with the Tziakas cocktail as described previously [28]. To detect $\mathrm{CaP}$ deposits, the VSMCs were immunostained with ARS and Calcein (Fig. 4). NR WT iPSC-derived VSMCs were not positive for ARS or Calcein following treatment with the calcification cocktail. R WT-, NR KO-, and $\mathrm{R}$ KO-derived VSMCs contained $\mathrm{CaP}$ deposits, as determined by positive ARS staining or Calcein staining (Fig. 4).

The $\mathrm{CaP}$ deposits were quantified with a python script based on Calcein staining (Suppl. 1). R WT-derived VSMCs showed a significant increase in $\mathrm{Ca}$ after treatment with the calcifying cocktail, while NR WT-derived VSMCs showed no change in Ca content (Suppl. Figure $8 \mathrm{~A}$ ). VSMCs derived from either the $\mathrm{R}$ or NR KO lines showed a similarly significant increase in Ca levels (Suppl. Figure 8B).

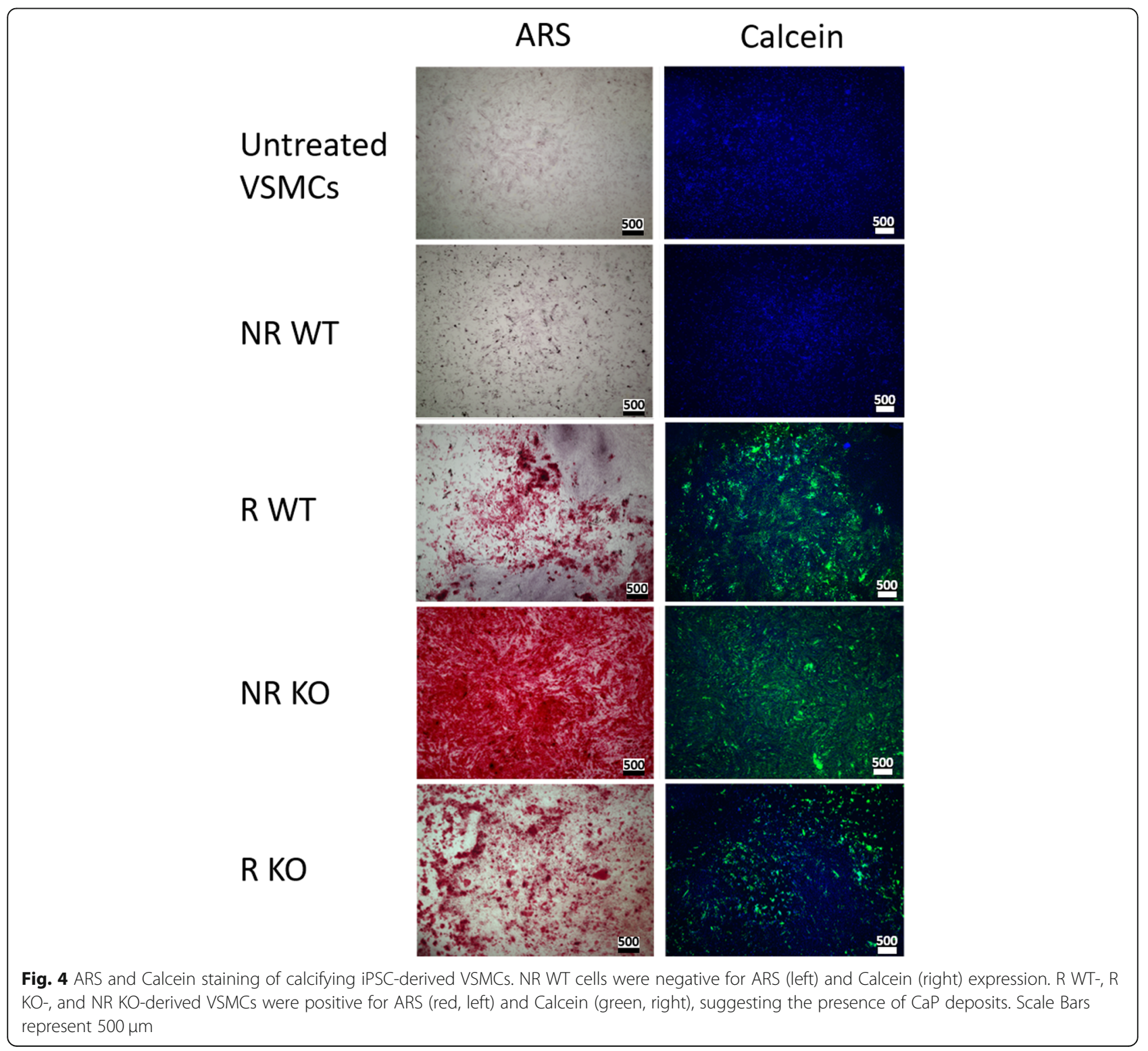




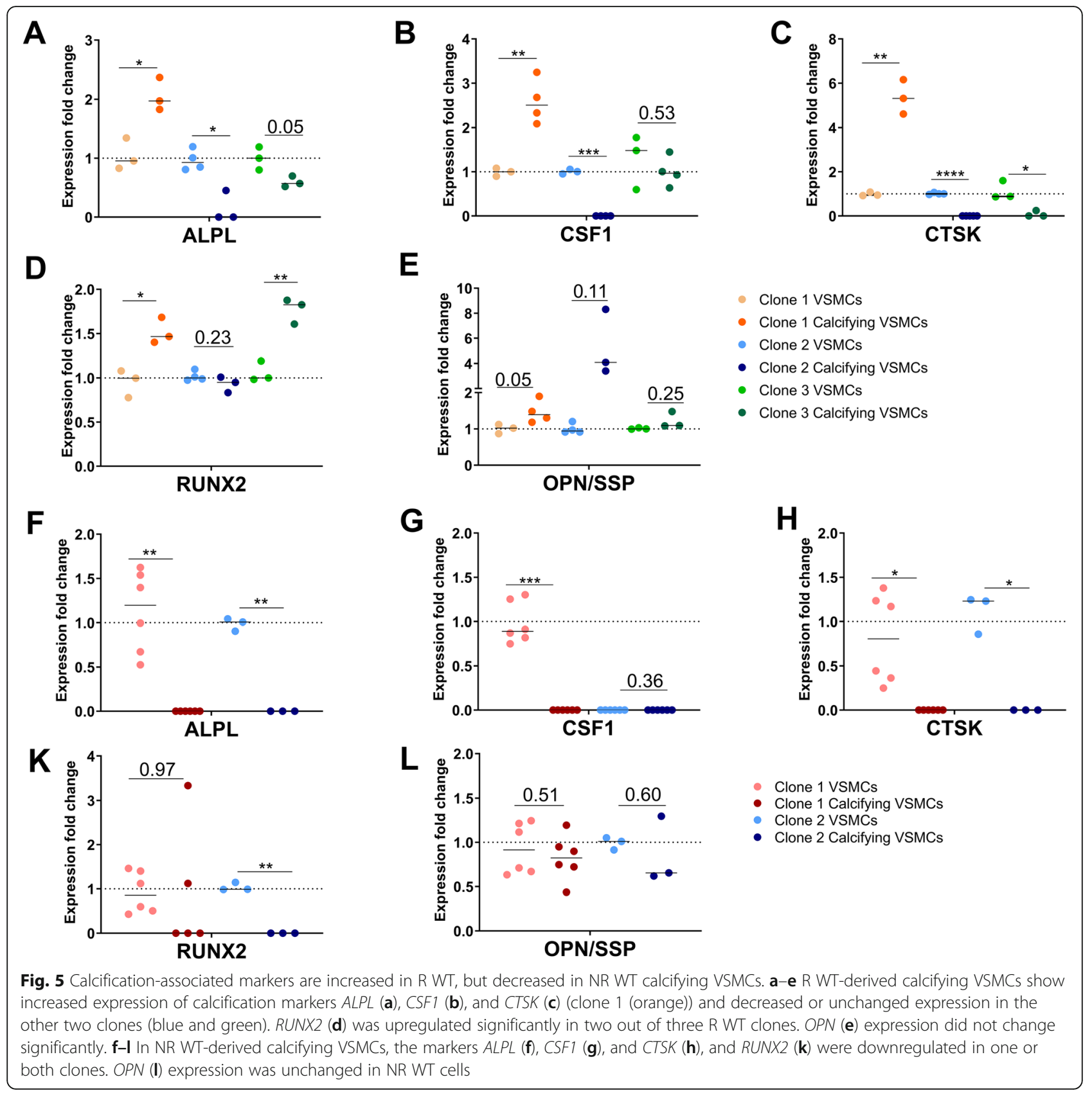

Expression of calcification-associated markers $A L P L$, CSF1, CTSK1, RUNX2, and OPN was investigated in calcifying VSMCs across all iPSC lines. Some genes (ALPL, $C S F 1$, or CTSK1) were upregulated in at least one clone of the R WT-derived VSMCs (Fig. 5). RUNX2 was upregulated in two of three clones (Fig. 5d), while OPN expression was unchanged in all three clones of the R WT genotype (Fig. 5e).

The expression profile of the NR WT iPSC-derived VSMCs (Fig. 5f-l) was more distinct. Most of the markers were downregulated in calcifying VSMCs compared with untreated VSMCs. Only OPN was unchanged in the calcifying VSMCs derived from both NR WT clones (Fig. 5l).

$\mathrm{R}$ KO-derived calcifying VSMCs showed significantly higher expression of ALPL (Fig. 6a), CSF1 (Fig. 6b), and RUNX2 than untreated VSMCs (Fig. 6d). Expression of CTSK (Fig. 6c) and OPN (Fig. 6e) was lower or similar in the $\mathrm{R}$ KO-derived VSMCs, whereas the genes CSF1 (Fig. 6g) and RUNX2 (Fig. $6 \mathrm{k}$ ) were upregulated in NR KO-derived calcifying VSMCs. The other markers were either downregulated or similarly expressed (Fig. 6f, h, and l).

Taken together, the gene expression patterns varied across the experiments and between genotypes. 


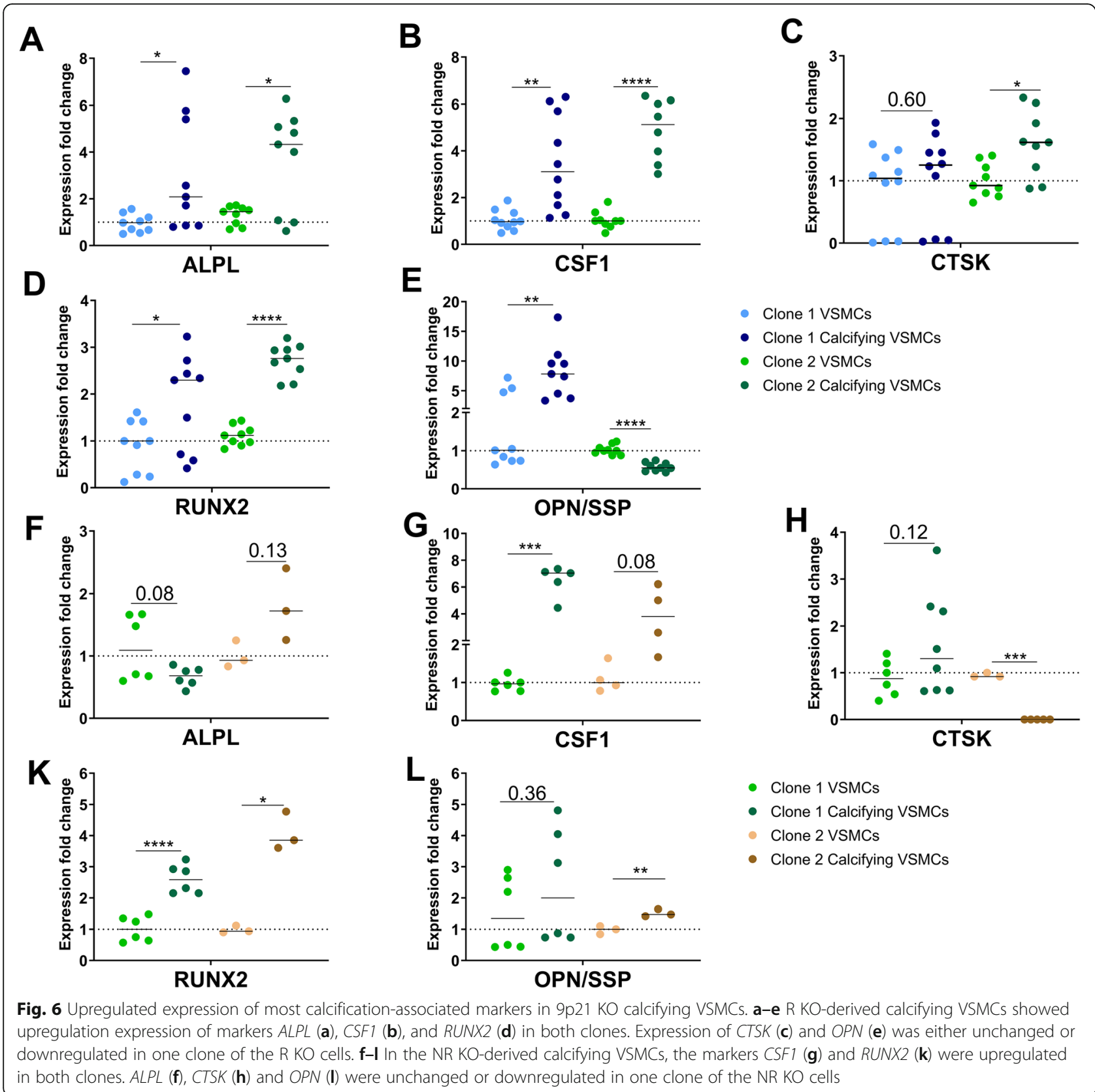

However, calcifying VSMCs derived from iPSCs with the 9p21 KO showed overall upregulation of most calcification markers. In conclusion, the NR WT-derived VSMCs did not calcify in response to the calcification stimulus. Meanwhile, calcifying VSMCs derived from the R WT, NR KO, and R KO iPSC lines showed signs of calcification and a partial increase in expression of calcification markers.

\section{Discussion}

Cardiovascular disease is the number one killer among all other diseases worldwide. GWAS have associated the 9p21 locus with CAD, myocardial infarction (MI), and vascular calcification $[7,30,31]$. In this study, we obtained human iPSCs from a healthy donor and a CAD risk donor carrying genetic variants within the $9 \mathrm{p} 21$ locus. Furthermore, iPSC lines with the 9p21 locus knocked-out were generated [27]. To study vascular calcification, VSMCs were differentiated from the iPSC lines and then calcified. To our knowledge, this is the first establishment of an in vitro calcification model.

iPSCs were differentiated into lateral mesodermderived VSMCs by slight modification of a previously published protocol [25]. No differences were observed 
between undifferentiated or VSMCs derived from either WT or KO iPSC lines. Across all cell lines, the pluripotency markers NANOG, OCT4, and SOX2, as well as the SMC-specific markers TAGLN, CNN1, and CALD1, were expressed in iPSCs and VSMCs, respectively. Therefore, the 9p21 locus does not seem to affect iPSC or VSMC gene expression. The proliferation and migration rates of iPSC-derived VSMCs were analyzed because they are relevant parameters for atherosclerosis. R WT and R KO-derived VSMCs showed a higher proliferation rate than those derived from NR WT and KO lines. This result supports a previous report [27] showing that proliferation is higher in R WT-derived VSMCs, but that KO of the $9 \mathrm{p} 21$ locus restores the proliferation rate to NR WT levels [27]. It could be assumed that the observed differences were due to the use of different techniques to determine the proliferation rate. For example, Lo Sardo et al. (2018) used the number of passages at a given time as an indication of proliferation rate. In the present study, we used Ki67 staining to quantify the number of proliferative cells relative to the total cell count (obtained using the nuclear stain DAPI). Both methods have their technical subtleties, which may lead to contradictory results.

The VSMCs were analyzed further using a scratch assay to measure migration rate. The assay revealed an increased migration rate in R WT-derived VSMCs compared with those derived from NR WT iPSCs. There was no significant difference between $\mathrm{R}$ KO- and NR KOderived VSMCs. Therefore, we were able to confirm that the 9p21 risk region influences VSMC behavior [27].

Moreover, the role of $9 \mathrm{p} 21$ in vascular calcification was assessed using a cellular-based assay. Previously, most in vitro calcification studies were conducted with either harvested mouse or human primary aortic SMCs or mesenchymal stem cells $[28,29]$. However, the use of primary cells is limited by ethics and accessibility and further compounded by differences between animal models and the human system.

For human iPSC-derived VSMCs, we used a previously published protocol consisting of a cocktail of $\mathrm{CaCl}_{2}, \beta$ GP, L-AP, insulin, and dexamethasone to generate an in vitro model of calcification [28]. This protocol was $44 \%$ efficient with respect to calcification of iPSCderived VSMCs from all cell lines (data not shown). NR WT-derived VSMCs showed no signs of calcification, neither by staining and quantification, nor by expression of associated markers ALPL, CTSK, CSF1, RUNX2, or $O P N$. R WT-, R KO-, and NR KO-derived VSMCs all contained $\mathrm{Ca}$ deposits, as determined by immunostaining and quantification analysis, with partial upregulation of calcification-associated markers. In conclusion, $\mathrm{KO}$ of the 9p21 CAD risk region leads to increased Ca deposition in VSMCs; therefore, patients carrying SNPs in this genomic locus are at greater risk for developing vascular calcification.

However, some variation was observed during the calcification experiments both between and within iPSC lines. The variable results may be due to the use of serum in the SMC maintenance and calcification media. Bovine serum contains anti-calcific agents such as fetuin-A, which decrease the calcification efficiency in vitro by up to $30 \%$ $[32,33]$. Furthermore, it is not clear what influence different matrices have, or which exact SMC phenotype is induced by the differentiation protocol used. In future, it would be desirable to optimize the protocol to improve efficiency and reduce variability.

Nevertheless, the method presented in this study provides a good basis for investigating vascular calcification of VSMCs in vitro using patient-derived iPSCs. In future, it could be applied to investigate other CAD gene candidates to estimate the heritable risk of vascular calcification. The underlying mechanisms leading to calcification of iPSC-derived VSMCs remain unclear and need to be investigated in future studies. In particular, the balance between linear versus circular ANRIL RNA and related pathways, as recently reported by Holdt and coworkers, would be worth investigating [34].

\section{Conclusion}

The 9p21 locus is associated with vascular calcification using GWAS. In this work, we aimed to go beyond GWAS and established a protocol to functionally study vascular calcification using iPSC-derived VSMC. We demonstrated increased proliferative and migratory ability of the 9p21 risk genotype in iPSC-derived VSMC, which are consistent with a recently published study. Furthermore, we demonstrated that the loss of or SNPs within the 9p21 CAD risk locus leads to increased vascular calcification in calcifying iPSC-derived VSMCs. Hence, patients carrying risk SNPs in the respective locus are of much higher risk of developing coronary calcification and thus coronary artery disease.

\section{Supplementary Information}

The online version contains supplementary material available at https://doi. org/10.1186/s13287-021-02229-5.

\footnotetext{
Additional file 1: Suppl. Figure 1. The 9p21 locus does not influence iPSC morphology or protein localization. Suppl. Figure 2. The 9p21 locus does not influence VSMC morphology or protein localization. Suppl. Figure 3. Representative images of Ki67 staining in R vs NR WT VSMCs. Suppl. Figure 4. Representative images of Ki67 staining in R vs NR WT VSMCs. Suppl. Figure 5. Representative images of migration of R vs NR WT VSMCs. Suppl. Figure 6. Representative images of migration of R vs NR KO VSMCs. Suppl. Figure 7. Efficiency of Tziakas calcification cocktail over genotypes. Suppl. 1. Python Script for Calcein quantification (by Dr. Tobias Reinberger). Suppl. Figure 8. Calcein quantifications had increased Ca levels in R WT, and both $\mathrm{KO}$ genotypes.
} 


\section{Abbreviations}

ARS: Alizarin Red Staining; CAC: Coronary artery calcification; $\mathrm{CaCl}_{2}$ : Calcium chloride; CAD: Coronary artery disease; CaP: Calcium phosphate; CDNA: Complementary DNA; DTT: Dithiotreitol; ECM: Extracellular matrix; GWAS: Genome-wide association study; IF: Immunofluorescence; iPSCs: Induced pluripotent stem cells; KO: Knockout; L-AP: L-Ascorbic acid phosphate; LM: Lateral mesoderm; MI: Myocardial infarction; NR: Non-risk; PFA: Paraformaldehyde; qPCR: Quantitative polymerase chain reaction; R: Risk; SMCs: Smooth muscle cells; VSMCs: Vascular smooth muscle cells; WT: Wildtype; $\beta$-GP: Beta-glycerophosphate

\section{Acknowledgements}

First, we would like to thank Prof Dr. Valentina Lo Sardo from University of Wisconsin-Madison, Prof. Dr. Kristin Baldwin and Prof. Dr. Ali Torkamani from the Scripps research institute, La Jolla, for kindly providing the 9p21 engineered iPS cells to us. We further thank Dr. Tobias Reinberger from University of Luebeck, Institute for Cardiogenetics, for the assistance with the Calcein quantification using Python. We further thank the Cardiogenetics members at University of Luebeck Sandra Wrobel, Annett Liebers, and Maren Behrensen, for their excellent technical assistance.

\section{Authors' contributions}

AT performed and analyzed the majority of the experiments, was involved in project design, and wrote the majority of the manuscript. MM and BS were involved in the maintenance of iPSCs and establishment of the differentiation protocol. UH contributed largely to the establishment of the differentiation and calcification protocols. JE and ZA contributed to the project design, editing and writing of the manuscript, and project funding. The authors read and approved the final manuscript.

\section{Funding}

This study was funded by Fondation Leducq (18CVD02, PlaqOmics). Open Access funding enabled and organized by Projekt DEAL.

\section{Availability of data and materials}

The data that support the findings of this study are available from the corresponding author upon reasonable request.

\section{Ethics approval and consent to participate}

Not applicable

\section{Consent for publication}

Not applicable

\section{Competing interests}

None

\section{Author details}

${ }^{1}$ Institute for Cardiogenetics, University of Luebeck, Ratzeburger Allee 160, 23562 Luebeck, Germany. ǱHK (German Centre for Cardiovascular Research), Partner Site Hamburg/Kiel/Luebeck, Luebeck, Germany. ${ }^{3}$ University Heart Centre Luebeck, 23562 Luebeck, Germany. ${ }^{4}$ Fraunhofer Institute for Molecular Biology and Applied Ecology (IME), 22525 Hamburg, Germany.

Received: 14 January 2021 Accepted: 14 February 2021

Published online: 06 March 2021

\section{References}

1. Nabel EG, Braunwald E. A tale of coronary artery disease and myocardial infarction. N Engl J Med. 2012;366(1):54-63. https://doi.org/10.1056/ NEJMra1112570.

2. Torpy JM, Burke AE, Glass RM. Coronary heart disease risk factors. JAMA. 2009;302(21):2388. https://doi.org/10.1001/jama.302.21.2388.

3. Wilson PWF, D'Agostino RB, Levy D, Belanger AM, Silbershatz H, Kannel WB. Prediction of coronary heart disease using risk factor categories. Circulation. 1998;97(18):1837-47. https://doi.org/10.1161/01.CIR.97.18.1837.

4. Marenberg ME, Risch N, Berkman LF, Floderus B, de Faire U. Genetic susceptibility to death from coronary heart disease in a study of twins. N Engl J Med. 1994;330(15):1041-6. https://doi.org/10.1056/NEJM1994041433 01503.
5. Myers RH, Kiely DK, Cupples LA, Kannel WB. Parental history is an independent risk factor for coronary artery disease: the Framingham study. Am Heart J. 1990; 120(4):963-9. https:/doi.org/10.1016/0002-8703(90)90216-K.

6. Erdmann J, Kessler T, Munoz Venegas L, Schunkert H. A decade of genomewide association studies for coronary artery disease: the challenges ahead. Cardiovasc Res. 2018;114(9):1241-57. https://doi.org/10.1093/cvr/cvy084.

7. Samani NJ, Erdmann J, Hall AS, et al. Genomewide association analysis of coronary artery disease. N Engl J Med. 2007;357(5):443-53. https://doi.org/1 0.1056/NEJMoa072366

8. Holdt LM, Teupser D. Recent studies of the human chromosome 9p21 locus, which is associated with atherosclerosis in human populations. Arterioscler Thromb Vasc Biol. 2012;32(2):196-206. https://doi.org/10.1161/A TVBAHA.111.232678.

9. McPherson R. Chromosome 9p21 and coronary artery disease. N Engl J Med. 2010:362(18):1736-7. https://doi.org/10.1056/NEJMcibr1002359.

10. Schunkert $H$, König IR, Kathiresan $S$, et al. Large-scale association analysis identifies 13 new susceptibility loci for coronary artery disease. Nat Genet. 2011;43(4):333-8. https://doi.org/10.1038/ng.784

11. Webb TR, Erdmann J, Stirrups KE, et al. Systematic evaluation of pleiotropy identifies 6 further loci associated with coronary artery disease. J Am Coll Cardiol. 2017:69(7):823-36. https://doi.org/10.1016/j.jacc.2016.11.056.

12. Jono $\mathrm{S}, \mathrm{McKee} M D$, Murry CE, et al. Phosphate regulation of vascular smooth muscle cell calcification. Circ Res. 2000;87(7):e10-7. https://doi.org/1 0.1161/01.RES.87.7.e10

13. Alam M, Kirton JP, Wilkinson FL, et al. Calcification is associated with loss of functional calcium-sensing receptor in vascular smooth muscle cells. Cardiovasc Res. 2009;81(2):260-8. https://doi.org/10.1093/cvr/cvn279.

14. Balderman JAF, Lee H-Y, Mahoney CE, et al. Bone morphogenetic protein-2 decreases microRNA-30b and microRNA-30c to promote vascular smooth muscle cell calcification. J Am Heart Assoc. 2012;1(6):e003905. https://doi. org/10.1161/JAHA.112.003905.

15. Byon $\mathrm{CH}$, Javed $\mathrm{A}$, Dai $\mathrm{Q}$, et al. Oxidative stress induces vascular calcification through modulation of the osteogenic transcription factor Runx2 by AKT signaling. J Biol Chem. 2008;283(22):15319-27. https://doi.org/10.1074/jbc M800021200.

16. Doherty TM, Asotra K, Fitzpatrick LA, et al. Calcification in atherosclerosis: bone biology and chronic inflammation at the arterial crossroads. Proc Natl Acad Sci U S A. 2003;100(20):11201-6. https://doi.org/10.1073/pnas.1932 554100.

17. Drake FH, Dodds RA, James IE, et al. Cathepsin K, but not cathepsins B, L, or S, is abundantly expressed in human osteoclasts. J Biol Chem. 1996;271(21): 12511-6. https://doi.org/10.1074/jbc.271.21.12511.

18. Giachelli CM. The emerging role of phosphate in vascular calcification. Kidney Int. 2009;75(9):890-7. https://doi.org/10.1038/ki.2008.644

19. Lutgens SPM, Cleutjens KBJM, Daemen MJAP, Heeneman S. Cathepsin cysteine proteases in cardiovascular disease. FASEB J. 2007;21(12):3029-41. https://doi.org/10.1096/fj.06-7924com.

20. Thompson B, Towler DA. Arterial calcification and bone physiology: role of the bone-vascular axis. Nat Rev Endocrinol. 2012;8(9):529-43. https://doi. org/10.1038/nrendo.2012.36.

21. Tintut $Y$, Alfonso $Z$, Saini $T$, et al. Multilineage potential of cells from the artery wall. Circulation. 2003;108(20):2505-10. https://doi.org/10.1161/01.CIR. 0000096485.64373. .C5.

22. Alexander MR, Owens GK. Epigenetic control of smooth muscle cell differentiation and phenotypic switching in vascular development and disease. Annu Rev Physiol. 2012;74(1):13-40. https://doi.org/10.1146/a nnurev-physiol-012110-142315.

23. Chen IY, Matsa E, Wu JC. Induced pluripotent stem cells: at the heart of cardiovascular precision medicine. Nat Rev Cardiol. 2016;13(6):333-49. https://doi.org/10.1038/nrcardio.2016.36.

24. Wobus AM, Boheler KR. Embryonic stem cells: prospects for developmental biology and cell therapy. Physiol Rev. 2005;85(2):635-78. https://doi.org/1 0.1152/physrev.00054.2003.

25. Cheung $C$, Bernardo AS, Pedersen RA, Sinha S. Directed differentiation of embryonic origin-specific vascular smooth muscle subtypes from human pluripotent stem cells. Nat Protoc. 2014;9(4):929-38. https://doi.org/10.1038/ nprot.2014.059.

26. Yang $L$, Geng Z, Nickel T, et al. Differentiation of human inducedpluripotent stem cells into smooth-muscle cells: two novel protocols. PLoS One. 2016;11(1):e0147155. https://doi.org/10.1371/journal.pone.0147155. 
27. Lo Sardo V, Chubukov P, Ferguson W, et al. Unveiling the role of the most impactful cardiovascular risk locus through haplotype editing. Cell. 2018. https://doi.org/10.1016/j.cell.2018.11.014

28. Tziakas DN, Georgios C, Maria P, et al. Lysed erythrocyte membranes promote vascular calcification. Circulation. 2019;139(17):2032-48. https://doi. org/10.1161/CIRCULATIONAHA.118.037166.

29. Alves RD, Eijken M, van de Peppel J, van Leeuwen JP. Calcifying vascular smooth muscle cells and osteoblasts: independent cell types exhibiting extracellular matrix and biomineralization-related mimicries. BMC Genomics. 2014;15(1). https://doi.org/10.1186/1471-2164-15-965.

30. Helgadottir A, Thorleifsson G, Manolescu A, et al. A common variant on chromosome 9p21 affects the risk of myocardial infarction. Science. 2007; 316(5830):1491-3. https://doi.org/10.1126/science.1142842.

31. McPherson R, Pertsemlidis A, Kavaslar N, Stewart A, Roberts R, Cox DR, Hinds DA, Pennacchio LA, Tybjaerg-Hansen A, Folsom AR, et al. A Common Allele on Chromosome 9 Associated with Coronary Heart Disease. Sci. 2007;316: 1488-91.

32. Holmar J, Noels H, Böhm M, Bhargava S, Jankowski J, Orth-Alampour S. Development, establishment and validation of in vitro and ex vivo assays of vascular calcification. Biochem Biophys Res Commun. 2020;530(2):462-70. https://doi.org/10.1016/j.bbrc.2020.05.085.

33. Reynolds JL, Joannides AJ, Skepper JN, et al. Human vascular smooth muscle cells undergo vesicle-mediated calcification in response to changes in extracellular calcium and phosphate concentrations: a potential mechanism for accelerated vascular calcification in ESRD. J Am Soc Nephrol JASN. 2004;15(11): 2857-67. https://doi.org/10.1097/01.ASN.0000141960.01035.28.

34. Holdt LM, Teupser D. Long noncoding RNA ANRIL: Lnc-ing genetic variation at the chromosome 9p21 locus to molecular mechanisms of atherosclerosis. Front Cardiovasc Med. 2018;5. https://doi.org/10.3389/fcvm.2018.00145.

\section{Publisher's Note}

Springer Nature remains neutral with regard to jurisdictional claims in published maps and institutional affiliations.

Ready to submit your research? Choose BMC and benefit from:

- fast, convenient online submission

- thorough peer review by experienced researchers in your field

- rapid publication on acceptance

- support for research data, including large and complex data types

- gold Open Access which fosters wider collaboration and increased citations

- maximum visibility for your research: over $100 \mathrm{M}$ website views per year

At $\mathrm{BMC}$, research is always in progress.

Learn more biomedcentral.com/submissions 